\title{
Strategies for the Development and Application of Research Frameworks in Sciences Education Research
}

\author{
Lindelani Mnguni \\ Office of Teaching and Learning, Faculty of Health Sciences, \\ University of the Witwatersrand, 1 Jan Smuts Ave., \\ Braamfontein 200o, Johannesburg, South Africa
}

DOI: https://doi.org/10.36941/jesr-2021-0123

\begin{abstract}
Recent research in social sciences and education shows that a significant number of studies are neither reproducible nor repeatable. This compromises the validity, reliability and trustworthiness of these studies, as they violate the prescriptions of the nature of science. This lack of validity, reliability and trustworthiness could be due to poorly conceptualized research frameworks, including the conceptual framework and theoretical framework. Additionally, there is an apparent confusion on the difference between the research frameworks and their role in research. The current paper defines the different research frameworks that are used in science education. It also provides systematic strategies for the development and application of research frameworks in science education research. By using these systematic strategies, researchers could enhance the validity, reliability and trustworthiness of their research.
\end{abstract}

Keywords: Contextual framework; Framework of constructs; Research frameworks; Science education; Theoretical framework

\section{Introduction}

Research scientists, including sciences education researchers, are generally regarded as custodians of science literacy. At the core of science literacy is the mastery of the nature of science through which the quality of scientific knowledge is enhanced. Perhaps the most critical tenet of the nature of science is that scientific knowledge is based on empirical evidence, which is generated through scientifically acceptable methods. These methods may be informed by various research frameworks and expressed through scientific models, laws, and theories through which natural phenomena are explained. A research framework is a theoretical structure which frames the research by informing the research plan and defining the exact set of activities in the research (Mill et al., 2010). A clearly defined research framework ensures that researchers follow scientifically acceptable methods in order to produce scientific knowledge that is valid and reliable.

Knekta et al. (2019) and Heale and Twycross (2015) define validity as the extent to which a research method measures what it is intended to. There are different forms of validity, including content validity, construct validity, face validity and criterion validity (Knekta et al., 2019). These are all intended to determine the validity of various components of research, including the methods, 
instruments, and data. Reliability refers to the extent to which a research method produces consistent results over time (Golafshani, 2003). In line with the nature of science, researchers rely on empirical evidence to demonstrate validity and reliability in their research. By establishing validity and reliability, researchers enhance the reproducibility and repeatability of their findings. Reproducibility refers to the degree of agreement produced by different researchers, at different locations using different instruments to answer the same research question (Goodman et al., 2016). It is related to repeatability, which refers to the degree of agreement produced by the same researcher using the same instruments at different times to answer the same research question (Vitek \& Kalibera, 2011). Reproducibility and repeatability are essential features of scientific research as they ensure that findings are logical, durable, objective and generalizable.

While validity and reliability are concepts generally associated with quantitative research, trustworthiness is associated with qualitative research. Given the possible subjectivity of qualitative researchers, trustworthiness "poses the question "can the findings be trusted?" (Korstjens \& Moser, 2018, p. 121). To determine trustworthiness, researchers explore credibility, dependability, transferability and confirmability (Patton, 1999). Credibility refers to the extent to which research methods can generate plausible findings. Dependability refers to the extent to which the research methods produce stable findings. Transferability refers to the extent to which the research findings could be transferred to another context with other respondents. Confirmability refers to the degree to which other researchers could confirm the findings.

\subsection{Poor research design and the quality of research}

In light of the above, it is critical for sciences education researchers to base their research on scientifically acceptable research frameworks, and methods in order to enhance the validity, reliability and trustworthiness of their research. Recent research in social sciences, including education, suggests that some researchers may be lacking an understanding of the research frameworks and methods. As a result, these researchers may be adopting poorly constructed and applied research frameworks, which in turn compromises the validity, reliability and trustworthiness of their research (Osanloo \& Grant, 2016). Iqbal (2007) reports that graduate students regard developing the research frameworks as the most challenging part of the research proposal. Similarly, Osanloo and Grant (2016) report that students express confusion and lack of knowledge with developing a research framework. Based on these views, therefore, the current paper aims to provide a clear definition of the research frameworks, as well as strategies of how they could be developed and applied in order to enhance the quality of research in sciences education. This is in line with Knekta et al.'s (2019, p. 2) argument that science education researchers ought to "produce more rigorous and replicable science".

The urgency for adopting well-established research methods is heightened by recent research which has cast doubt on the quality of some educational and social sciences research. In particular, research has shown that several studies in social sciences cannot be replicated (Dreber \& Johannesson, 2019), lacks reliability (Pashler \& Wagenmakers, 2012) and is not reproducible (Camerer et al., 2018; Simmons et al., 2011). Researchers have attributed the "replication crisis" mainly to poor research methods (Dreber \& Johannesson (2019; Pashler \& Wagenmakers, 2012). Academic pressure has also been identified as causing researchers to 'pressure cook' their studies in order to publish (Simmons et al., 2011). This pressure cooking of research may be particularly true concerning research frameworks, such as theoretical frameworks, where only the product is presented, but the methods used to generate these frameworks are rarely presented. As such, research frameworks are one component of education research which are likely to compromise the quality of research.

While the "replication crisis" has not been reported in science education, researchers in the field must take extra precautions to ensure that their research adheres to the scientific principles. As such, attempts (such as Warfa, 2016) to enhance researchers' understanding of research methods in sciences education should be encouraged. This is because "(science) education research will move 
forward if the (science) community more fully understands the specific methodological issues encountered by individuals working at the intersection of science and education. For example, what do social scientists mean by "theory" and "theoretical framing," and how can learning theories and other relevant knowledge be used to inform the design and interpretation of (science) education research?" (Dolan \& Stone, 2013).

The current essay addresses the research frameworks as a critical component of education research. In particular, it will attempt to distinguish the different frameworks in education research, and provide strategies on how these could be developed and used to enhance the validity, reliability and trustworthiness of research. This could enhance science education researchers' theoretical understanding of research frameworks and help in ensuring that these are employed appropriately in the science education research context (Knekta et al., 2019).

\section{The Frameworks in Education Research}

\subsection{Framework of constructs}

The first framework in education research is the conceptual framework. Maxwell (2012) proposes that a conceptual framework is "the system of concepts, assumptions, expectations, beliefs, and theories that supports and informs" research. Imenda (2014) suggests that a conceptual framework "could then be used in place of a theoretical framework. Thus, a conceptual framework may be defined as a result of bringing together several related concepts to explain or predict a given event, or give a broader understanding of the phenomenon of interest - or simply, of a research problem". The problem with Maxwell's (2012) and Imenda's (2014) propositions, however, is that they have a potential of creating confusion about the difference between the conceptual framework and the theoretical framework. Confusion may be caused by the fact that Maxwell's definition implies that a conceptual framework is made up of theories that support research. At the same time, Imenda's states that a conceptual framework can be used in place of a theoretical framework. As such, they both imply that the conceptual framework and the theoretical framework are similar. To resolve this confusion, it is essential to provide a clear definition for each of these, paying particular attention to their uniqueness.

A conceptual framework is derived from the term 'scientific concept' which is defined as the generally accepted collection of meanings or characteristics associated with specific scientific phenomena. Liehr and Smith (1999, p. 7) suggest that a concept "is an image or symbolic representation" of an abstract scientific idea. Similarly, Chinn and Kramer (1999, p. 252) state that concepts are "complex mental formulation of experience" which "convey the abstract ideas within a theory". Leggett (2011) contends, however, that concepts have progressive levels of abstraction. She argues that abstract concepts are scientifically called constructs because a conceptual definition provides meaning to a construct in abstract or theoretical terms. This allows abstract concepts to be made concrete so that they can be studied. She defines a construct as an image or abstract idea invented explicitly for a given research and theory-building purpose (Leggett, 2011). This suggests that constructs are conceptualized within a specific research context as they are "heavily theory laden" and "show the interrelationship between the key concepts" (Van der Walt, 2020, p. 2). Within a research context, constructs may have a conceptual meaning and an operational meaning. A conceptual meaning provides meaning to one construct in abstract or theoretical terms, while the operational meaning specifies the procedures used to measure a construct (Leggett, 2011).

Given the definition of a construct, the current author posits that researchers may use literature to conceptualize constructs by providing context-specific meaning to specific constructs. Additionally, researchers may indicate how these constructs will be operationalized in their research. 
The conceptualization and operationalization of constructs lead to the development of a framework of constructs, in which researchers provide a detailed account of how they generated the constructs, as well as how these constructs will be used in their research. A framework of constructs, therefore, is a conceptualization and operationalization of constructs in a research study. It is a logical reproducible model of concepts, and variables in a research context, which defines the behaviour, nature and characteristics of these constructs within a specific research context. The purpose of a framework of constructs is to provide standard conceptual and operational meanings which are to be adopted in a research context.

\subsection{A theoretical framework}

The second framework in education research is the theoretical framework, which is made up of a single or multiple theories. Kerlinger and Lee (2000, p. 11) define a theory as "a set of interrelated constructs (concepts), definitions, and propositions that present a systematic view of phenomena by specifying relations among variables, to explain and predict phenomena." Emerging from Kerlinger and Lee's (2000) definition is that a theory is used to explain the behaviour of constructs in a research study and can be used to explore and explain scientific phenomena. To this, scholars suggest that a theoretical framework is a blueprint which explains how theory will inform the exploration and explanation of constructs in a research study (Adom et al., 2018; Imenda, 2014; Osanloo \& Grant, 2016; Rocco \& Plakhotnik, 2009). Therefore, it follows that the theoretical framework should inform the characterization of constructs in a research study. According to Kivunja (2018, p. 45), this is because:

- $\quad$ "a theory is a set of propositions consisting of defined and interrelated constructs;

- a theory sets out the interrelations among a set of variables (constructs), and in so doing, presents a systematic view of the phenomena described by the variables; and,

- a theory explains phenomena; it does so by specifying which variables are related to which variables and how they are related, thus enabling the researcher to predict from certain variables to certain other variables".

Given the above, a theoretical framework can be defined as a logical model of theories, through which constructs are characterized and described within the parameters of the composite theories. The purpose of the theoretical framework is to explain aspects of phenomena that can be researched through accepted scientific methods. It can also be used to determine protocols of observations, measurement and inform the interpretation of results in order to deduce findings.

\subsection{Contextual framework}

A third research framework is the contextual framework, which is derived from a survey of scholarly work as an in-depth synthesis of existing knowledge about a particular topic. This synthesis is generally referred to as the literature review. It explores substantive findings in the field as well as theoretical and methodological contributions to a particular topic. The challenge, however, with defining this as the literature review is that, technically, a majority of a scientific research study entails some degree of literature review. This includes a literature review to identify a research gap, provide a rationale for the research, identify a research paradigm and research methods and discuss research findings. Consequently, the current author suggests that instead of a 'literature review', researchers ought to provide a contextual framework.

A contextual framework can be defined as a distinctive review, critique, and conceptualization of existing knowledge through which a hypothesis and thesis statement are synthesized. In the contextual framework, a researcher may critically explore corroborations, gaps, contradictions and discrepancies in literature as an attempt to provide a context-specific convergent framework of what is known, what needs to be investigated, why it needs to be investigated and how it needs to be investigated in a selected research area. Literature survey in developing a contextual framework, 
ranges from being "exhaustive, to exhaustive with selective citation (i.e., the entire literature is examined, but only selected pieces of literature are discussed in detail), to representative, to central or pivotal (i.e., only literature that is influential, highly original, controversial, or provocative is reviewed)" (Torraco, 2016, p. 405). Additionally, a contextual framework may be organized "conceptually, such that literature addressing the same ideas is reviewed together; historically, according to the chronological order in which issues appeared in the literature; or, methodologically, in which literature using the same research methods are reviewed together" (Torraco, 2016, p. 405).

\section{Strategies for Developing Research Frameworks}

The process of developing any of the three research frameworks can be inductive or deductive (Figure 1). Imenda (2014) suggests that the process of developing a research framework may be inductive where the researcher surveys literature, identifies individual components and then pieces them together to generate a map of relations between the individual components. In the case of the framework of constructs, these components would be the individual constructs, while in the theoretical framework, they would be the individual theories. In the contextual framework, the individual components could include previous studies that explored similar phenomena in various contexts (e.g., different countries, and socio-economic contexts). This means the researcher, having identified individual components of the framework, would seek patterns from existing literature and then develop theoretical explanations from these patterns (Bernard, 2017). Through inductive reasoning, the researcher may use the developed research framework to make inferences about possible outcomes within a specific research context (Romeyn, 2004). In this case, the purpose of the research would be to test the veracity of the research framework. As such, the research framework would inform the research questions, research methods, the data to be collected, and how these data are to be analyzed and interpreted.
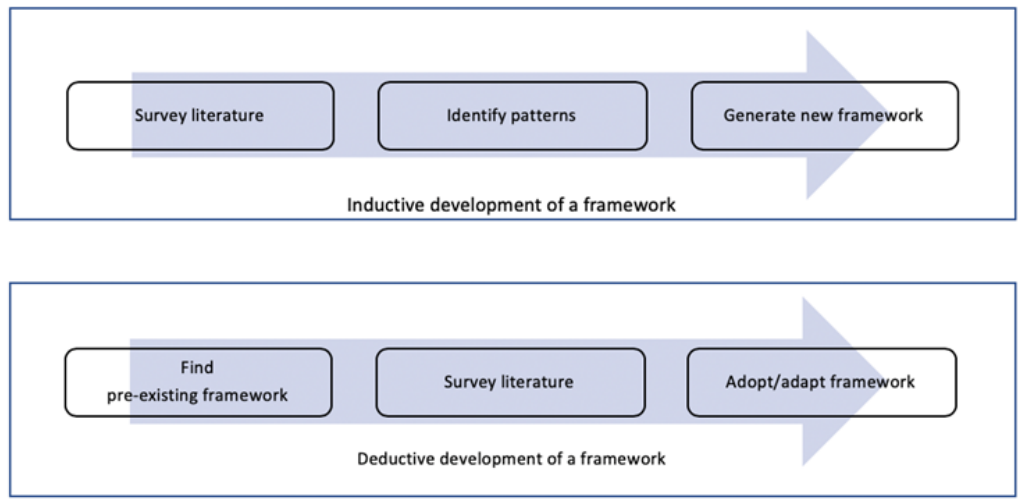

Figure 1: Procedures for developing a research framework inductively and deductively.

In a deductive approach, a researcher develops a hypothesis based on existing theory and then designs a research strategy to test the hypothesis (Wilson, 2010). Within the context of developing a research framework, this means, the researcher would first identify a pre-existing research framework from literature, which may have been used in similar research studies. After that, the researcher would conduct a systematic literature survey through which the veracity of this existing research framework could be verified. The emerging literature may also be used to adapt or adopt the research framework.

When developing a research framework using the inductive and deductive methods, the 
researcher ought to follow a systematic method of literature survey. As suggested by Dewey and Drahota (2016), in a systematic literature survey, the researcher identifies, selects and critically appraises literature as a way of responding to a specific literature review aim. This suggests that, at the beginning of the process, the researcher must explicitly state the aim of the literature survey. During the survey, the researcher must follow a clearly defined protocol where criteria for literature to be surveyed is stated. In such a protocol, the researcher may state the survey strategy in terms of key terms, search strategies, sources of literature, as well as the analysis strategies employed. The purpose of such a detailed process is to enhance the replicability and reproducibility of the literature survey itself, which in itself should conform to the nature of science.

\section{Application of Research Frameworks}

Having developed the framework of constructs, theoretical framework and the contextual framework, the researcher would then proceed to contribute new knowledge into the research framework which may corroborate, or contradict existing knowledge or provide new insights (Figure 2). In this regard, research may be used to test a hypothesis or build a hypothesis for further research, which is essentially the fundamental purpose of research. This means the fundamental aim of the research study should reflect the contribution concerning the research framework.

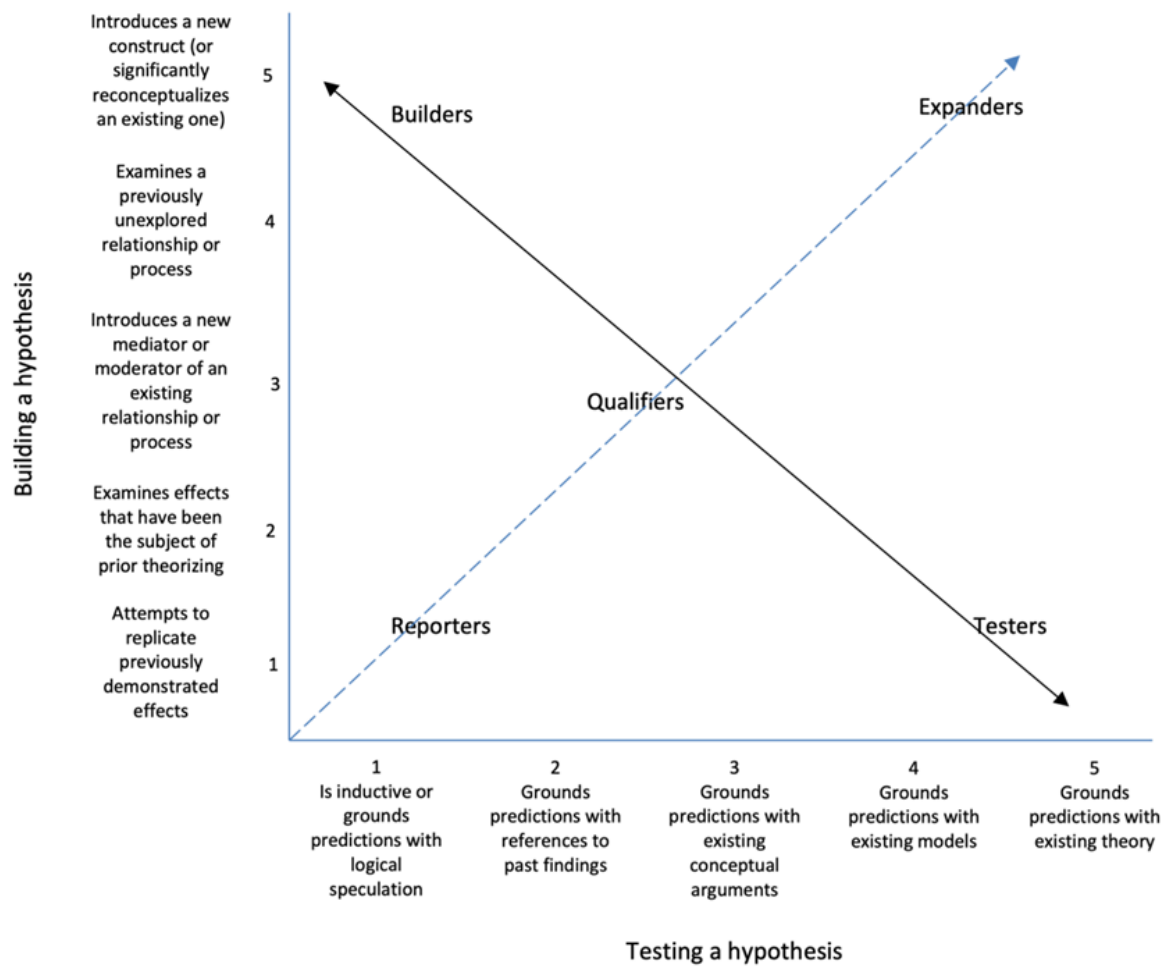

Figure 2: A taxonomy of theoretical contributions for empirical research (adapted from Colquitt \& Zapata-Phelan, 2007)

Testing a hypothesis refers to a research process where data are collected and analyzed to determine whether there is evidence that supports a given hypothesis (Hak \& Dul, 2009a). For example, having 
developed a research framework, a researcher may explore empirical evidence to determine whether data from a specific research context support the hypothesis. Alternatively, a researcher may use logical speculations, past findings, existing conceptual argument, existing models or other existing theories to test a hypothesis (Figure 2, Colquitt \& Zapata-Phelan, 2007). For example, in the case of a theoretical framework, the researcher may use empirical evidence to accept a hypothesis, to reject it, or to propose modifications. In the case of a contextual framework, empirical evidence may be used to corroborate, or contradict existing knowledge, or provide novel insights into a phenomenon. The framework of constructs may also be used to determine the ideal variables, including sources of data, sample size, type of data to be collected, and variables to be manipulated or studied. Ultimately, research frameworks should form an integral part of a research study.

Depending on the research context, a researcher may use a single case or multiple cases to test a hypothesis. The number of cases may depend on the underlying research methodology, including the research paradigm. Quantitative methods may be used if the researcher adopts a positivist paradigm, in which case multiple cases (or relatively large sample size) may be used for data collection. In line with the requirements of quantitative research, large sample sizes enhance reliability. If the researcher adopts an interpretivist/constructivist paradigm, then a single case (or smaller sample size) may be used where qualitative data are collected.

Building a hypothesis refers to the process of using a research framework to propose an explanation of a phenomenon (Kamasak et al., 2017). According to Eisenhardt and Graebner (2007, p. 25) this "involves using one or more cases to create theoretical constructs, propositions and/or midrange theory from case-based, empirical evidence". Consequently, the hypothesis being built could be a new contribution to a theoretical framework or a new contribution to an existing contextual framework. The fundamental aim in this regard is to propose new insights regarding the nature, character or behaviour of constructs. This can be done by replicating previously demonstrated effects of constructs, examining effects of constructs that have been subject of theoretical perspectives, introducing new constructs, or exploring the new relationship between constructs (Figure 2, Colquitt \& Zapata-Phelan, 2007).

Hak and Dul (2009b) suggest that research that seeks to build a hypothesis requires sample cases through which the new propositions can be tested. However, Wacker (1998) proposes that in building a hypothesis, researchers must first provide definitions of the constructs which are to be included in the framework. These definitions may emerge from an existing framework of constructs which is generated through a literature survey. Once the constructs have been defined, detailed observations are described within predetermined cases (Hak \& Dul, 2009b). The objective in this regard is to describe the behaviour of constructs within these cases. Such descriptions could be made through descriptive quantitative research methods or qualitative designs such as ethnography, phenomenological study, case study or grounded theory. Following case data analysis, the researcher may proceed to explore cross-case patterns to determine whether observations made within cases exist between cases (Hak \& Dul, 2009b). If patterns are determined, the researcher may then seek to replicate his or her observation within and between cases as a way of verifying "that the emergent propositions fit with the evidence in each case" (Hak \& Dul, 2009b, p. 339). Eisenhardt and Graebner (2007) suggest that central to building research frameworks from cases is the replication logic, where individual cases are seen as distinct experiments of analytic units. By replicating case findings, researchers seek to enhance validity, reliability and trustworthiness of their findings. Replication is also critical in terms of the nature of science, which requires that scientific findings should be replicable, repeatable, reproducible and reliable. As such, researchers building a new hypothesis are obligated to provide evidence of replicability (Hak \& Dul, 2009b).

\section{Conclusion}

Based on emerging discourses in literature, the current author is of the view that research may be regarded as invalid, unreliable and untrustworthy due to several aspects other than the research 
methods, results and findings. This, therefore, calls for the adoption of a systematic method in the development of all research components, including the research frameworks. The current paper has provided a clear definition for each of the research frameworks, which could help researchers in conceptualizing their research. Additionally, in the current paper, the author has provided some strategies which could be used in the development and application of these frameworks. The author believes that by using these strategies; researchers will now be able to follow a systematic method when conceptualizing the frameworks of their research, which could enhance the validity, reliability and trustworthiness of their research.

\section{References}

Adom, D., Hussein, E. K., \& Agyem, J. A. (2018). Theoretical and conceptual framework: Mandatory ingredients of a quality research. International Journal of Scientific Research, 7(1), 438-441.

Bernard, H. R. (2017). Research methods in anthropology: Qualitative and quantitative approaches. Rowman \& Littlefield. Camerer, C. F., Dreber, A., Holzmeister, F., Ho, T. H., Huber, J., Johannesson, M., Kirchler, M., Nave, G., Nosek, B. A., Pfeiffer, T., Altmejd, A., Buttrick, N., Chan, T., Chen, Y., Forsell, E., Gampa, A., Heikensten, E., Hummer, L., Imai, T., . . Wu, H. (2018). Evaluating the replicability of social science experiments in Nature and Science between 2010 and 2015. Nature Human Behaviour, 2(9), 637-644. https://doi.org/10.1038/s41562-018-0399-z

Chinn, P. L., \& Kramer, M. K. (1999). Theory and Nursing: A Systematic Approach (5th ed.). Mosby.

Colquitt, J. A., \& Zapata-Phelan, C. P. (2007). Trends in theory building and theory testing: A five-decade study of the Academy of Management Journal. Academy of Management Journal,50(6), 1281-1303. http://www.jstor.com/stable/20159472https://doi.org/10.5465/amj.2007.28165855

Dewey, A., \& Drahota, A. (2016) Introduction to systematic reviews: online learning module. Cochrane Training. https://training.cochrane.org/interactivelearning/module-1-introduction-conducting-systematic-reviews

Dolan, E. L., \& Stone, E. (2013). Adding to the biology education research tool kit: research methods essays. CBELife Sciences Education, 12(3), 320-321. https://dx.doi.org/10.1187/cbe.13-06-0109

Dreber, A., \& Johannesson, M. (2019). Statistical significance and the replication crisis in the social sciences. In Oxford Research Encyclopedia of Economics and Finance. https://dx.doi.org/10.1093/acrefore/9780190625979.013.461

Eisenhardt, K. M., \& Graebner, M. E. (2007). Theory building from cases: Opportunities and challenges. Academy of Management Journal, 50(1), 25-32. http://www.jstor.com/stable/20159839 https://doi.org/10.5465/amj.2007.24160888

Golafshani, N. (2003). Understanding reliability and validity in qualitative research. Qualitative Report, 8(4), 5976o7. http://www.nova.edu/ssss/QR/QR8-4/golafshani.pdf

Goodman, S. N., Fanelli, D., \& Ioannidis, J. P. (2016). What does research reproducibility mean? Science Translational Medicine, 8(341), 341ps12-341ps12. https://www.doi.org/10.1126/scitranslmed.aaf5027

Hak, T., \& Dul, J. (2009a). Theory-testing with cases. In A. J. Mills, G. Durepos, \& E. Wiebe (Eds.), Encyclopedia of Case Study Research (pp. 937-942). Sage.

Hak, T., \& Dul, J. (2009b). Theory-building with cases. In A. J. Mills, G. Durepos, \& E. Wiebe (Eds.), Encyclopedia of Case Study Research (pp. 932-937). Sage.

Heale, R., \& Twycross, A. (2015). Validity and reliability in quantitative studies. Evidence-Based Nursing, 18(3), 6667. https://doi.org/10.1136/eb-2015-102129

Imenda, S. (2014). Is there a conceptual difference between theoretical and conceptual frameworks? Journal of Social Sciences, 38(2), 185-195. https://www.doi.org/10.108o/o9718923.2014.11893249

Iqbal, J. (2007). Learning from a Doctoral Research Project: Structure and Content of a Research Proposal. Electronic Journal of Business Research Methods, 5(1), 11-20.

Kamasak, R., Kar, A., Yavuz, M., \& Baykut, S. (2017). Qualitative Methods in Organizational Research: An Example of Grounded Theory Data Analysis. In B. Christiansen \& H. C. Chandan (Eds.), Handbook of Research on Organizational Culture and Diversity in the Modern Workforce (pp. 23-42). IGI Global., https://doi.org/10.4018/978-1-5225-2250-8.choo2

Kerlinger, F. N. \& Lee, H. B. (200o). Foundations of behavioural research. 4th Ed. Belmont, CA: Cengage Learning.

Kivunja, C. (2018). Distinguishing between theory, theoretical framework, and conceptual framework: A systematic review of lessons from the field. International Journal of Higher Education, 7(6), 44-53. https://doi.org/10.5430/ijhe.v7n6p44

Knekta, E., Runyon, C., \& Eddy, S. (2019). One size doesn't fit all: Using factor analysis to gather validity evidence when using surveys in your research. CBE-Life Sciences Education, 18(1), rm1. https://dx.doi.org/10.1187/cbe.18-04-0o64 
Korstjens, I., \& Moser, A. (2018). Series: Practical guidance to qualitative research. Part 4: Trustworthiness and publishing. The European Journal of General Practice, 24(1), $120-124$. https://www.doi.org/10.1080/13814788.2017.1375092

Leggett, A. (2011). Constructs, variables and operationalization. In J. F. Hair, R. P. Bush, \& D. J. Ortinau (Eds.), Marketing Research within a Changing Information Environment (pp. 56-93). McGraw-Hill.

Liehr, P., \& Smith, M. J. (1999). Middle range theory: Spinning research and practice to create knowledge for the new millennium. Advances in Nursing Science, 21(4), 81-91. https://doi.org/10.1097/ooo12272-199906000-00011

Maxwell, J. A. (2012). Qualitative research design: An interactive approach (3rd ed.). Sage publications.

Mills, A. J., Durepos, G., \& Wiebe, E. (2010). Sage encyclopedia of case study research. Sage. https://dx.doi.org/10.4135/9781412957397.n299

Osanloo, A., \& Grant, C. (2016). Understanding, selecting, and integrating a theoretical framework in dissertation research: Creating the blueprint for your "house". Administrative Issues Journal: Connecting Education, Practice, and Research, 4(2), 7. https://www.doi.org/10.5929/2014.4.2.9

Pashler, H., \& Wagenmakers, E. J. (2012). Editors' introduction to the special section on replicability in psychological science: A crisis of confidence? Perspectives on Psychological Science, 7(6), 528-530. https://doi.org/10.1177/1745691612465253

Patton, M. Q. (1999). Enhancing the quality and credibility of qualitative analysis. Health Services Research, 34(5 Pt 2), 1189-1208. https://www.ncbi.nlm.nih.gov/pmc/articles/PMC1089059/pdf/hsresearchooo22-0112.pdf

Rocco, T. S., \& Plakhotnik, M. S. (2009). Literature reviews, conceptual frameworks, and theoretical frameworks: Terms, functions, and distinctions. Human Resource Development Review, 8(1), $120-130$. https://doi.org/10.1177/1534484309332617

Romeyn, J. W. (2004). Hypotheses and inductive predictions. Synthese, 141(3), 333-364. https://doi.org/10.1023/B:SYNT.ooooo44993.82886.9e

Simmons, J. P., Nelson, L. D., \& Simonsohn, U. (2011). False-positive psychology: Undisclosed flexibility in data collection and analysis allows presenting anything as significant. Psychological Science, 22(11), $1359-1366$. https://doi.org/10.1177/o956797611417632

Torraco, R. J. (2016). Writing integrative literature reviews: Using the past and present to explore the future. Human resource development review, 15(4), 404-428. https://doi.org/10.1177/1534484316671606

Van der Walt, G. (2020). Constructing conceptual frameworks in social science research. T.D.The Journal for Transdisciplinary Research in Southern Africa, 16(1), 1-9. https://doi.org/10.4102/td.v16i1.758

Vitek, J., \& Kalibera, T. (2011, October). Repeatability, reproducibility and rigor in systems research. In 2011 Proceedings of the Ninth ACM International Conference on Embedded Software (EMSOFT) (pp. 33-38). IEEE. https://doi.org/10.1145/2038642.2038650

Wacker, J. G. (1998). A definition of theory: research guidelines for different theory-building research methods in operations management. Journal of Operations Management, 16. 361 - 385. https://doi.org/10.1016/So272$6963(98) 00019-9$

Warfa, A. R. M. (2016). Mixed-methods design in biology education research: Approach and uses. CBE-Life Sciences Education, 15(4), rm5. https://doi.org/10.1187/cbe.16-01-0022.

Wilson, J. (2010). Essentials of Business Research: A Guide to Doing Your Research Project. SAGE Publications. 